\title{
EARTHQUAKE RESPONSE ANALYSES OF SOIL-STRUCTURE INTERACTION SYSTEM USING NONLINEAR ENERGY TRANSMITTING BOUNDARY
}

\author{
Naohiro NAKAMURA ${ }^{1}$ \\ ${ }^{1}$ Research \& Development Institute, Takenaka Corporation, \\ 1-5-1, Ohtsuka, Inzai, Chiba, Japan, 270-1395 \\ nakamura.naohiro@takenaka.co.jp
}

Keywords: Transmitting boundary, Frequency Dependency, Nonlinear, Soil Impedance, Time History Response Analysis.

\begin{abstract}
The energy-transmitting boundary, which is used in the well-known FEM program FLUSH, is a quite efficient technique for the earthquake response analysis of buildings considering soil-structure interaction. However, it is applicable only in the frequency domain. The author has studied and proposed methods for transforming frequency dependent soil impedance into the time domain.

In the previous paper, the author proposed an earthquake response analysis method using the energy transmitting boundary in the time domain using the transform method, because the kernel of the boundary is the frequency dependent impedance full matrix. Then, an earthquake response analysis with a nonlinear building using the boundary was carried out, and the accuracy and the efficiency of the boundary were confirmed. In that analysis, the inner field (the region inside the boundary) could be treated as the nonlinear system, but the free field (the region outside the boundary) and the boundary were treated as the linear system.

Contrary to this, in this paper, the nonlinear transmitting boundary is proposed. By using $i t$, all of the inner field, the free field and the boundary itself can be treated as the nonlinear system. The boundary impedance matrix is calculated in the frequency domain corresponding to the condition of the free field at the specific times. Then, these impedance matrices are transformed to the impulse response matrices in the time domain. In the nonlinear response analysis of the total system, the transmitting boundary at each time step is obtained by interpolation of these impulse response matrices between the specific times. Then, example earthquake response analyses were performed using a practical soil and building model to evaluate the soil-structure interaction effect. The response accuracy was compared with the cases of viscous boundary, which is the most common and representative boundary in the time domain. It was shown that the area of the inner field can be greatly reduced by using the proposed method because the accuracy of the boundary is quite high. Therefore, it was confirmed that the proposed method is effective.
\end{abstract}




\section{INTRODUCTION}

The energy transmitting boundary (hereinafter referred to as TB), which is used in FLUSH [1] and ALUSH [2], is a side wave boundary that is highly accurate and is highly effective. These programs developed in the 1970s are still utilized today as powerful tools in design study for architecture and civil engineering. However, TB has been formulated in the frequency domain, and can only perform linear analysis and equivalent linear analysis within the frequency domain. During a severe earthquake the building and its surrounding soil exhibit nonlinear behaviour. It is thus desirable to be able to use this boundary in the time domain also.

The author has previously studied how to transform TB to the time domain with high accuracy and ease in cases where the dynamic stiffness has a strong frequency dependency [3, 4]. As part of these efforts, the author transformed TB of the 2-dimensional in-plane problem, which is equivalent to FLUSH, into the time domain, and showed that high-accuracy analysis is possible in the same manner as with the frequency domain. In addition, the author applied a nonlinear characteristic to an inner field building, conducted response analysis, and demonstrated the effectiveness of this method for nonlinear problems [5] (hereinafter referred to as "the previous study").

In the above study, it was possible to consider the nonlinear characteristic for the inner field, but it was necessary to treat the outer field (free field) and TB as linear or equivalent linear. In this paper, the author makes it possible to consider the nonlinear characteristic for outer field objects as well as TB, and proposes a seismic response analysis method that considers the entire analysis model as nonlinear (See Table 1). The analysis target is a 2dimensional in-plane problem, in the same manner as the previous study.

First, the author outlines the evaluation method for the nonlinear characteristic of outer field, inner field, and TB. Due to the nonlinear characteristics of the material, the physical properties of the outer field changes at each time step. TB also changes at each time step based on the outer field change. However, if we constantly recalculate TB, the computational load will likely become significant. Therefore, in this paper the author proposes a method to interpolate TB which was calculated at representative time. Furthermore, as an example study, the author conduct a seismic response analysis of a soil-structure interaction system (hereafter referred to as SSI system) using these nonlinear analysis methods, thus demonstrating the effectiveness of the nonlinear TB. For comparison, the viscous boundary [6] (hereafter referred to as VB), which is the conventional representative method, and linear TB used in the previous study are used.

\begin{tabular}{|l|c|c|c|}
\hline & $\begin{array}{c}\text { Outer } \\
\text { field }\end{array}$ & TB & $\begin{array}{c}\text { Inner } \\
\text { field }\end{array}$ \\
\hline Frequency Domain TB & L & L & L \\
\hline Time Domain TB & L & L & N \\
\hline Proposed Method & N & N & N \\
\hline
\end{tabular}

*) L: linear or equivalent linear, N: nonlinear

Table 1 Method proposed in this paper

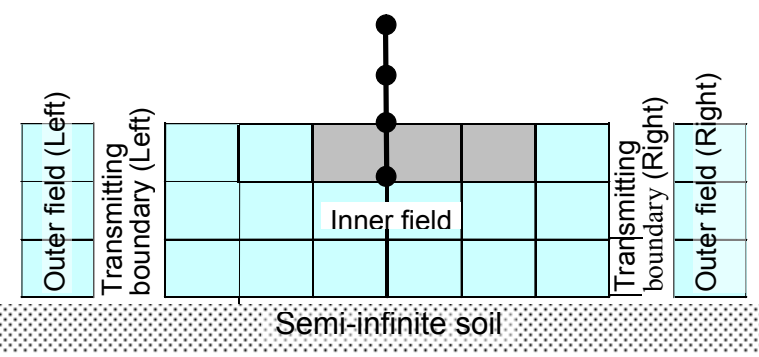

Figure 1 Image of analysis model studied in this paper

\section{ANALYSIS METHOD USING NONLINEAR TB}

In seismic response analysis which uses $\mathrm{TB}$, the analysis region can be divided into three sections, as shown in Figure 1: (1) outer field, (2) inner field, (3) TB. In this chapter, the au- 
thor outlines the method of nonlinear response analysis used in this paper for each of these three sections.

\subsection{Outer field}

The outer field is distant from the inner field and represents parallel layered soil (free field) which has sufficient spread. In order to be consistent with the analysis result of the inner field, a 1-dimensional wave propagation analysis is performed using a vertical column soil model in which the depth direction is split and soil physical properties the same as the outermost edge of the inner field. This is achieved by constraining the vertical degrees of freedom such that only horizontal shear deformation is allowed.

In the previous study, linear analysis was performed for the analysis of the outer field using 1-dimensional wave theory (SHAKE [7]). In this paper, nonlinear analysis is performed using the same plane strain elements as the outermost edge of the inner field. Physical properties of each element are considered to change based on the given dynamic deformation characteristics (G- $\gamma, h-\gamma$ relationship). Here, $G$ is the shear modulus, $h$ is the damping ratio, and $\gamma$ is the shear strain.

In this paper, nonlinear causal hysteretic damping model [8] is used for the soil analysis. This is a model where the stiffness and damping changes at each time step under the condition that the damping ratio is almost constant in a certain frequency range. Shear modulus and damping ratio changes at each time step depending on the maximum shear strain of the previous 1 second. It should be noted that while Reference [8] uses the lumped mass model with shear element, this paper uses the plane strain element. However, there is no essential difference in the analysis.

\subsection{Inner Field}

The inner field consists of the building, the foundation, and soil in the vicinity thereof. In this paper, the building is represented by a lumped mass model with shear element. The restoring force characteristics are set for each member, and time history nonlinear responses analysis is performed.

The soil, as with the outer field, is modeled using plane strain elements, and the nonlinear characteristic of every moment is evaluated by the nonlinear causal hysteretic damping model, based on the given G- $\gamma$, h- $\gamma$ relationship. For the shear strain, in the same manner as FLUSH, the main shear strain $\left(\gamma_{1}\right)$ of Equation (1) is used, and the shear modulus and damping ratio are varied according to the maximum principal shear strain $\left(\gamma_{1 \max }\right)$ of the previous 1 second. In addition, in Equation (1), $\varepsilon_{\mathrm{x}}, \varepsilon_{\mathrm{y}}, \gamma_{\mathrm{xy}}$ are respectively $\mathrm{x}$ direction axis strain, y direction axis strain, xy direction shear strain.

$$
\gamma_{1}=\sqrt{\left(\varepsilon_{x}-\varepsilon_{y}\right)^{2}+\gamma_{x y}^{2}}
$$

For this analysis, as with FLUSH, the Poisson's ratio does not change even after nonlinear deformation. As a result, the Young's modulus of each element changes with the same ratio as the shear modulus.

\section{$2.3 \mathrm{~TB}$}

TB is also changed depending on the change of the physical properties of outer field. However, calculating the impedance matrix of TB for each $\Delta \mathrm{T}$ (analysis time step) is likely to incur a large computational load. Therefore, for a certain time interval (hereinafter referred to as 
$\Delta \mathrm{T}_{\mathrm{b}}$ ), TB is calculated using the physical properties of that time, and is also interpolated during that time. More specifically, the calculation is performed as follows (see Figure 2):

(1) First the outer field is calculated, the soil physical properties are determined for each time $\Delta \mathrm{T}_{\mathrm{b}}\left(0, \Delta \mathrm{T}_{\mathrm{b}}, 2 \Delta \mathrm{T}_{\mathrm{b}}, 3 \Delta \mathrm{T}_{\mathrm{b}}, \ldots\right)$.

(2) Using this soil physical property, the impedance matrix of $\mathrm{TB}$ for each $\Delta \mathrm{T}_{\mathrm{b}}$ is calculated.

(3) The components of the matrix for each $\Delta \mathrm{Tb}$ are transformed to the time domain, and the impulse response matrix is calculated. In addition, the time domain transform method is $\mathrm{C}$ method, which is the same as the previous study.

(4) The parameters that control the interpolation of TB are selected. In this paper, the element which has the maximum shear strain in the outer field is selected, and the shear strain value of that element at each time is used as the control parameter (hereinafter, $\gamma_{\text {Emax }}(\mathrm{t})$ ).

(5) Let us assume, current time (hereinafter referred to as t) is between $\mathrm{j} \Delta \mathrm{T}_{\mathrm{b}}$ and $(\mathrm{j}+1) \Delta \mathrm{T}_{\mathrm{b}}$. First, we solve the ratio that $\gamma_{E \max }(\mathrm{t})$, and internally divide $\gamma_{\mathrm{Emax}}\left(\mathrm{j} \Delta \mathrm{T}_{\mathrm{b}}\right)$ and $\gamma_{\mathrm{Emax}}$ $\left((j+1) \Delta T_{b}\right)$. The impulse response matrix at time $t$ has the same ratio as above, and is used to internally divide the impulse response matrices of $\mathrm{j} \Delta \mathrm{T}_{\mathrm{b}}$ and $(\mathrm{j}+1) \Delta \mathrm{T}_{\mathrm{b}}$. The impulse response matrices for all analysis times are thereby calculated.

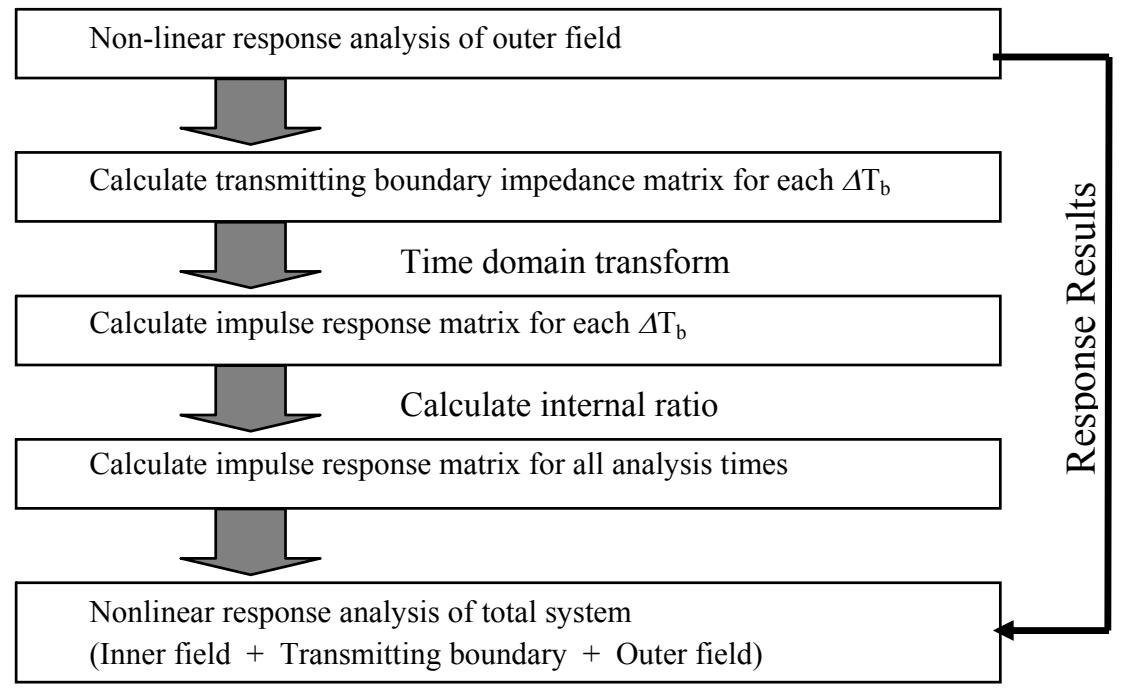

Figure 2 Flow chart of earthquake response analysis using non-linear transmitting

In this paper, $\Delta \mathrm{T}_{\mathrm{b}}$ is set to 1 second and $\Delta \mathrm{T}$ is set to 0.01 seconds. If $\Delta \mathrm{T}_{\mathrm{b}}$ is decreased to $\Delta \mathrm{T}$, it becomes the same as calculating TB matrix at each analysis time.

\subsection{Equation of motion of the time domain}

Equation of motion of the time domain basically is the same as in the previous study. However, in the study, the equation of motion was formulated as a $1 / 2$ model, using inversesymmetry conditions. In this paper, however, it is formulated as a full model without using an inverse symmetry condition to account for nonlinear characteristics, and a TB and free field are provided to the left and right of the model. In addition, the material of the inner field and TB are nonlinear.

Considering these factors, the equation of motion is given by equation (2). Here $\{\mathrm{u}(\mathrm{t})\}$ is the displacement vector, $\left[\mathrm{M}_{\mathrm{I}}\right],\left[\mathrm{K}_{\mathrm{I}}(\mathrm{t})\right]$ are a mass matrix and a stiffness matrix of the inner field, respectively. Also $[\mathrm{L}(\mathrm{t})],[\mathrm{R}(\mathrm{t})]$ are $\mathrm{TB}$ matrix on the left and right sides, and $\left\{\mathrm{F}_{\mathrm{L}}(\mathrm{t})\right\}$, 
$\left\{\mathrm{F}_{\mathrm{R}}(\mathrm{t})\right\}$ are the boundary force vectors on the left and right sides. The stiffness matrix and $\mathrm{TB}$ matrix are nonlinear, so $(\mathrm{t})$ is attached to indicate change with time.

$$
\begin{aligned}
& {\left[M_{I}\right]\{\ddot{u}(t)\}+\left(\left[K_{I}(t)\right]+[L(t)]+[R(t)]\right)\{u(t)\}=-\ddot{y}(t)\left[M_{I}\right]\{1\}+\left\{F_{L}(t)\right\}+\left\{F_{R}(t)\right\}} \\
& \left\{F_{L}(t)\right\}=\left([L(t)]-\left[D_{L}(t)\right]\right)\left\{u_{L}^{*}(t)\right\}, \quad\left\{F_{R}(t)\right\}=\left([R(t)]-\left[D_{R}(t)\right]\right)\left\{u_{R}^{*}(t)\right\}
\end{aligned}
$$

Where:

$$
\left[D_{R}\right]_{j}=-\left[D_{L}\right]_{j}=\frac{1}{2}\left[\begin{array}{cccc}
0 & \lambda(t)_{j} & 0 & -\lambda(t)_{j} \\
G(t)_{j} & 0 & -G(t)_{j} & 0 \\
0 & \lambda(t)_{j} & 0 & -\lambda(t)_{j} \\
G(t)_{j} & 0 & -G(t)_{j} & 0
\end{array}\right]
$$

$\left\{\mathrm{u}_{\mathrm{L}} *(\mathrm{t})\right\}$ and $\left\{\mathrm{u}_{\mathrm{R}} *(\mathrm{t})\right\}$ of the Equation (3) are the response displacement of the right and left side free field which are calculated by nonlinear analysis of the outer field. In addition, $\left[\mathrm{D}_{\mathrm{L}}(\mathrm{t})\right]\left\{\mathrm{u}_{\mathrm{L}} *(\mathrm{t})\right\}$ and $-\left[\mathrm{D}_{\mathrm{R}}(\mathrm{t})\right]\left\{\mathrm{u}_{\mathrm{R}} *(\mathrm{t})\right\}$ are the correction force vector (also known as excavation force) acting on the left boundary and right boundary when the earthquake motion is propagated from downward in the vertical direction.

$\left[D_{L}(t)\right]$ and $\left[D_{R}(t)\right]$ are represented by the superposition of sub-matrix $\left[D_{L}(t)\right]_{j}$ and $\left[D_{R}(t)\right]_{j}$ which are given by Equation (4), using the Lame constant $(\mathrm{G}(\mathrm{t}) \mathrm{j}, \lambda(\mathrm{t}) \mathrm{j})$ of each element of the free field. Since the Lame constants of each element change over time, $\left[D_{L}(t)\right]$ and $\left[D_{R}(t)\right]$ also change over time. In addition, when performing the equivalent linear analysis, $[L(t)],[R(t)]$, $\left[D_{L}(t)\right],\left[D_{R}(t)\right]$ are constant values which do not change over time.

Among these, $[\mathrm{L}(\mathrm{t})],[\mathrm{R}(\mathrm{t})]$ are time domain transformed values of the impedance which has a frequency dependency. For this reason, for the calculation of $[\mathrm{L}(\mathrm{t})]\{\mathrm{u}(\mathrm{t})\}$, in addition to the current displacement vector, the current velocity and acceleration vectors and the past displacement and velocity vectors are needed. The same is true for the calculation of $[\mathrm{R}(\mathrm{t})]\{\mathrm{u}(\mathrm{t})\}$, $[\mathrm{L}(\mathrm{t})]\left\{\mathrm{u}_{\mathrm{L}} *(\mathrm{t})\right\},[\mathrm{R}(\mathrm{t})]\left\{\mathrm{u}_{\mathrm{R}} *(\mathrm{t})\right\}$. For more information please refer to the previous study.

\subsection{Nonlinear causal hysteretic damping model}

In time history earthquake response analysis of the inner field and outer field, the causal hysteretic damping model is used in the same manner as in the previous study, as a way to represent the frequency-independence of the material damping. The applicability of causal hysteretic damping model to the nonlinear element has been confirmed in Reference 13. In the example analysis of the next chapter, it is used for the quadrilateral plane strain elements representing the soil, and shear spring elements representing the building.

Equation (5) shows the relational expression of element displacement - element force of the time domain using the nonlinear causal hysteretic damping model. It is almost the same as the previous study. In this paper, however, to take into account the nonlinear characteristics of the element, time change is represented by $(t)$ attached to the element stiffness matrix $\left[\mathrm{K}_{\mathrm{e}}(\mathrm{t})\right]$ and element damping ratio $h_{\mathrm{e}}(\mathrm{t})$.

$$
\left\{F_{e}(t)\right\}=\left[K_{e}(t)\right]\left[\left\{u_{e}(t)\right\}+2 h_{e}(t)\left(c_{0}\left\{\dot{u}_{e}(t)\right\}+\sum_{j=1}^{n} k_{j}\left\{u_{e}\left(t-t_{j}\right)\right\}\right)\right]
$$


Also $\left\{\mathrm{F}_{\mathrm{e}}\right\},\left\{\mathrm{u}_{\mathrm{e}}\right\}$ are the element force vector and the element displacement vector, respectively. Damping force is calculated by the damping term simultaneous component $\left(\mathrm{c}_{0}\right)$ and the stiffness term time delay component $\left(\mathrm{k}_{1}, \mathrm{k}_{2}, \ldots \mathrm{k}_{\mathrm{n}}\right)$, where $\left.\mathrm{k}_{\mathrm{j}}=\mathrm{k}(\mathrm{j} \Delta \mathrm{t})\right)$. As indicated in the previous study, the study frequency range is set as $0-10 \mathrm{~Hz}, \Delta \mathrm{t}=0.05 \mathrm{~s}$, and the 18 term model [9] is used.

\section{EXAMPLE STUDY}

In this chapter, an example problem is studied to evaluate the applicability and effectiveness of the proposed nonlinear TB.

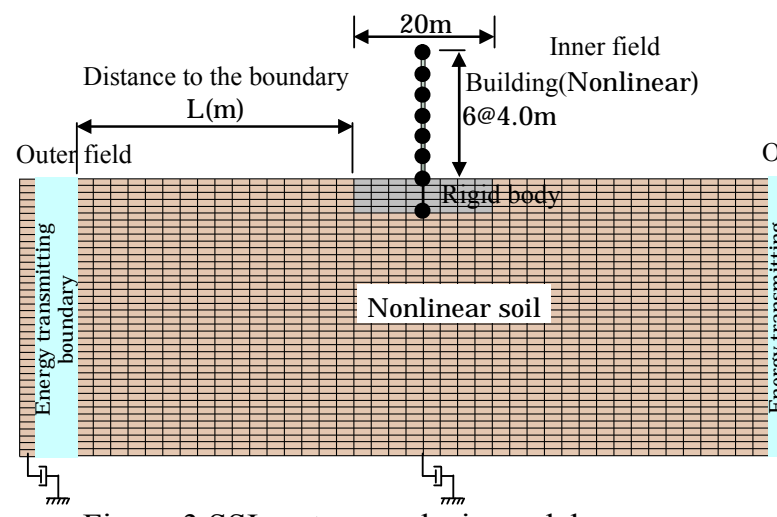

Figure 3 SSI system analysis model

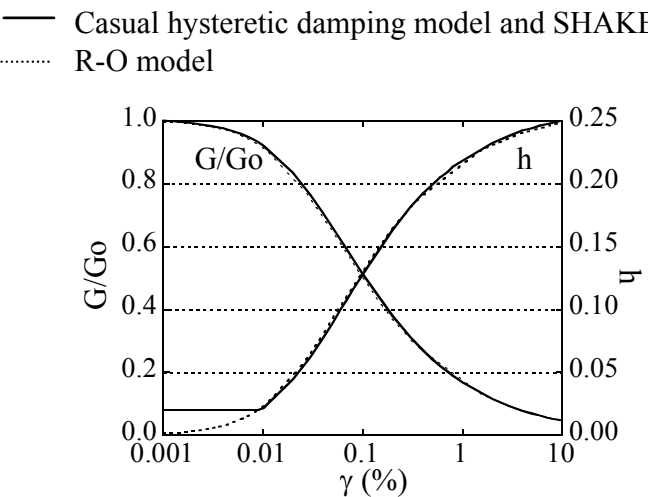

Figure 4 Dynamic deformation characteristics of soil

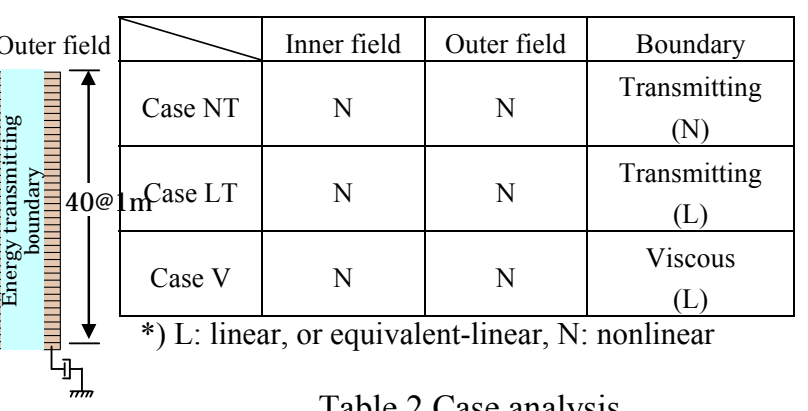

Table 2 Case analysis

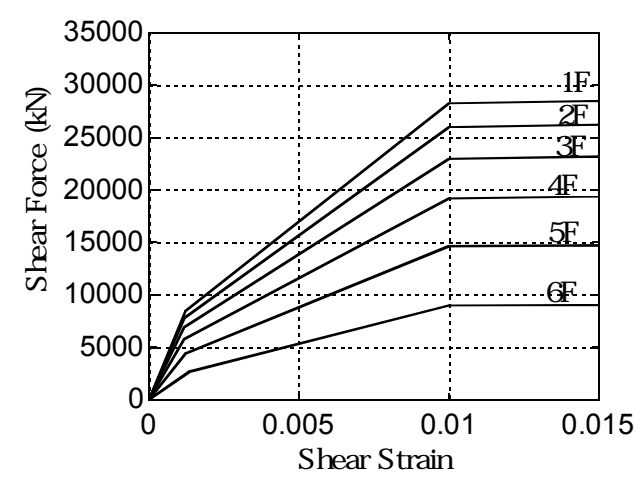

Figure 5 Building skeleton curves

\subsection{Analysis model and analysis condition}

The analysis model is shown in Figure 3. Both the soil and building have nonlinear characteristics. Soil consists of the surface layer and the base rock. In the surface layer, the thickness is $40 \mathrm{~m}$, the shear velocity is $300 \mathrm{~m} / \mathrm{s}$, and the nonlinear characteristics are considered based on the G- $\gamma$, h- $\gamma$ relationship shown in Figure 4 . As for the base rock, shear velocity is $500 \mathrm{~m} / \mathrm{s}$, and the effect is considered as the bottom VB. Figure 5 shows the skeleton curve of each floor of the building. The shear force of the first folding point and second folding point of each curve have been set to correspond to the static seismic intensity $0.3,1.0$ respectively.

Input earthquake motion is defined as $2 \mathrm{E}$ (twice the upward wave) using maximum acceleration 500Gal of El Centro $1940 \mathrm{NS}$ wave (duration: 10 seconds, time step $\Delta \mathrm{T}=0.01$ se- 
conds). Time integration is carried out using the Newmark- $\beta$ method and the average acceleration method $(\beta=1 / 4)$.

\subsection{Calculation of the outer field}

First, the time history nonlinear analysis of the outer field was performed. In this paper, the nonlinear causal hysteretic damping model $\left(\mathrm{F}_{\max }=10 \mathrm{~Hz}, 18\right.$ term model $)$ is used. For each soil element, calculation is performed using the shear modulus and damping ratio determined from the given G- $\gamma$, h- $\gamma$ relationship, for the maximum shear strain amplitude absolute value of the past $\Delta \mathrm{t}_{\mathrm{m}}$ seconds. Hereinafter, $\Delta \mathrm{t}_{\mathrm{m}}$ is called the maximum strain memory time.

First, we look at the impact the difference of $\Delta t_{m}$ has on response. The distribution of the maximum response values of acceleration, shear strain, and shear stress when $\Delta \mathrm{tm}$ is $0.1 \mathrm{~s}$, $0.5 \mathrm{~s}, 1.0 \mathrm{~s}, 2.0 \mathrm{~s}$ are shown in Figure 6 . From these figures, the result of $\Delta \mathrm{t}_{\mathrm{m}}=0.1 \mathrm{~s}$ shows a difference from others, but the other results roughly correspond. Thus, the result of $\Delta \mathrm{t}_{\mathrm{m}}=1.0 \mathrm{~s}$ is used in the subsequent study.
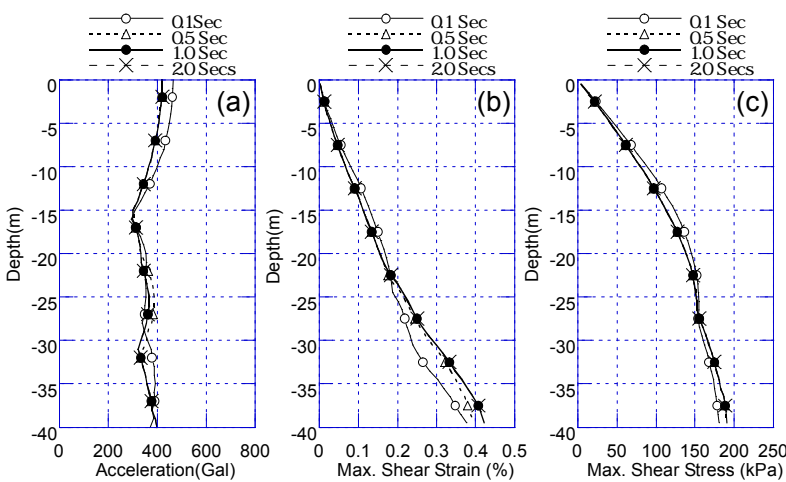

Figure 6 Distribution of maximum response of outer field (Effect of differences in $\Delta t_{m}$ on response), (a) Maximum acceleration, (b) Maximum shear strain, and (c) Maximum shear stress.
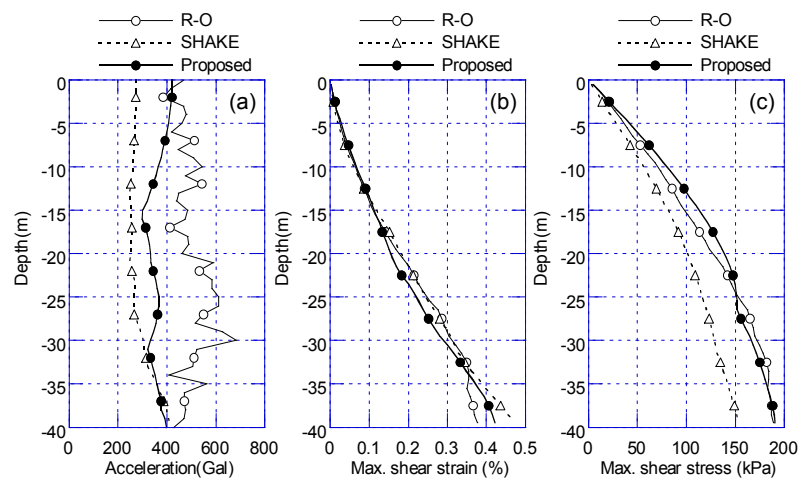

Figure 7 Distribution of maximum response of outer field (Comparison of R-O and SHAKE) (a) Maximum acceleration, (b) Maximum shear strain, and (c) Maximum shear stress.

Next, nonlinear analysis using the R-O model, and equivalent linear analysis using SHAKE is performed, and compared with the causal hysteretic damping model $\left(\Delta \mathrm{t}_{\mathrm{m}}=1.0 \mathrm{~s}\right)$. Figure 7 shows the maximum response value distribution of acceleration, shear strain, and shear stress. The shear strain reaches a maximum at the lowest element and was $0.42 \%$ in the causal hysteretic damping model, $0.38 \%$ in the R-O model, and $0.47 \%$ in SHAKE. The results of each model corresponds well overall, However, results of the R-O model for maximum acceleration has a discontinuous change and has some difference with others. This behavior is similar to the results shown in reference [9].

In this analysis, the shear wave velocity (Vs) and damping ratio (h) of each element is needed for each time for the later calculation of TB. For this reason, the later study is performed using the nonlinear causal hysteretic damping model in which these values are explicitly output.

\subsection{Calculation of TB}

The calculation of the nonlinear TB is performed based on the response results of the outer field by the causal hysteretic damping model. The number of DOF (degrees of freedom) of TB becomes 82 ( 41 nodes $\times 2$, i.e., horizontal and vertical degrees of freedom). In addition, using the results of the equivalent linear analysis of the outer field according to SHAKE, VB 
and linear TB of the time domain are also calculated (see Table 2). Calculation of the nonlinear TB is performed as follows in accordance with the flowchart in Figure 2.

Figure 8 indicates the shear strain time history waveform of the representative position of the outer field. As shown in the previous section, $\Delta \mathrm{t}_{\mathrm{m}}=1.0 \mathrm{~s}$ is used. According to $\gamma$ of $\Delta \mathrm{t}_{\mathrm{m}}=1.0$ $\mathrm{s}$, the shear modulus $\mathrm{G}$ and the damping ratio $h$ changes based on the $\mathrm{G}-\gamma, \mathrm{h}-\gamma$ relationship. TB matrix of the frequency domain is calculated using the physical properties at each $\Delta T_{b}$. In this paper, $\Delta \mathrm{T}_{\mathrm{b}}=1 \mathrm{~s}$ is used.

Figure 9 shows the distribution of the shear strain, Vs (shear wave velocity), and h (damping ratio) calculated from the G- $\gamma$, h- $\gamma$ relationship at representative times $(1 \mathrm{~s}, 3 \mathrm{~s}, 8 \mathrm{~s})$,. Accordingly, TB matrix of the frequency domain corresponding to the soil physical properties is calculated for each time. Frequency analysis carried out over the range of $0.5 \mathrm{~Hz}$ to $20 \mathrm{~Hz}$ in $0.5 \mathrm{~Hz}$ increments. From here, TB matrix is obtained as a frequency-dependent complex matrix of $82 \times 82$ components, for each time.
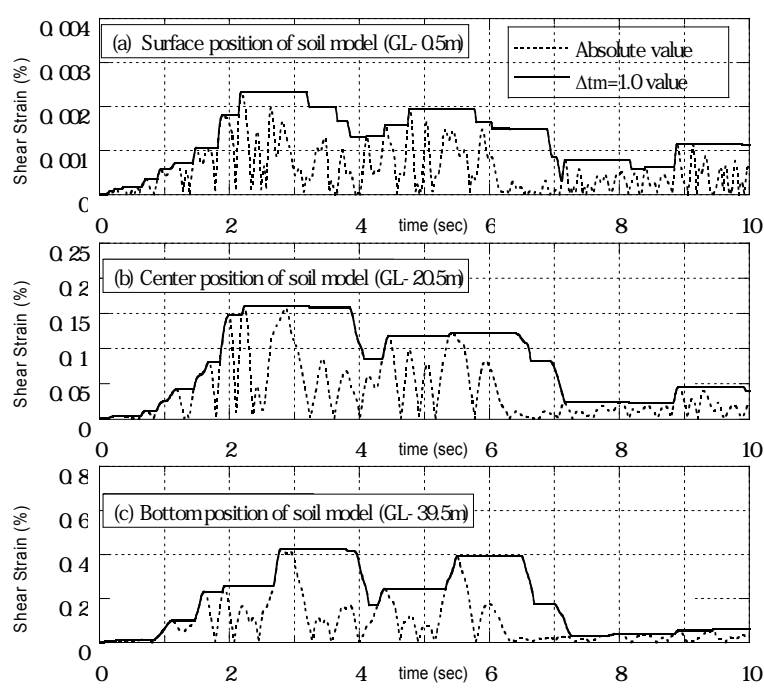

Figure 8 Shear strain time history waveform of the representative position of the outer field
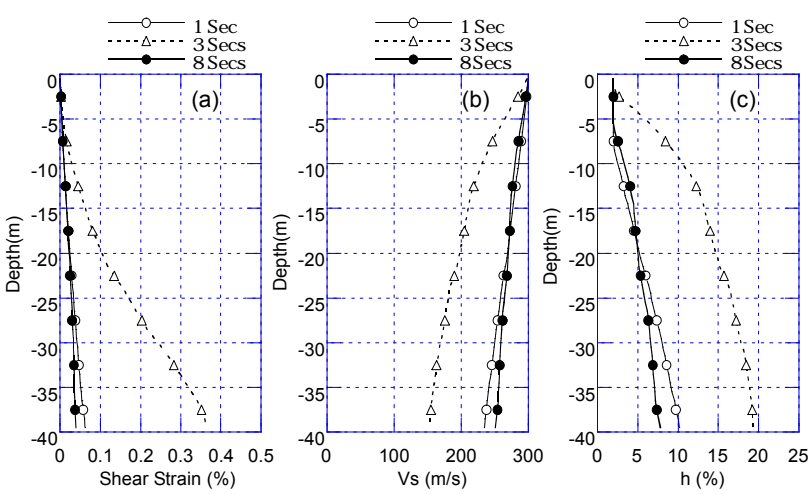

Figure 9 Representative time distribution, (a) Shear Strain, (b) Shear wave velocity, and (c) damping ratio

These impedance matrices are transformed to the time domain, and TB impulse response matrix for each $\Delta \mathrm{T}_{\mathrm{b}}$ is obtained. The method to transform time domain, is the $\mathrm{C}$ method, and the analysis conditions are the same as in the previous study.

By interpolating TB impulse response matrix for each $\Delta \mathrm{T}_{\mathrm{b}}$ from the previous section, the impulse response matrix for each analysis time step ( $\Delta \mathrm{T}$ : 0.01 seconds in this study) is calculated. The control point used for interpolation is the shear strain of the element of the bottom layer that produces maximum strain.

\subsection{SSI system response analysis}

The nonlinear soil and building are set in the inner field, and a SSI system response analysis is performed. To clarify the impact that the differences in the boundary have on response, the outer fields are all unified in nonlinear analysis.

The study is performed for the three cases indicated in Table 2. Case NT uses the nonlinear TB proposed in this paper. Case LT uses the equivalent linear TB proposed in the previous study. Case V uses a VB representative of a conventional method. In Case LT and Case V, equivalent linear analysis of the outer field using SHAKE was performed, and TB and VB 
calculated from the equivalent physical properties obtained. Furthermore, the effect of the width of the inner field (L) on the accuracy is investigated for each case.
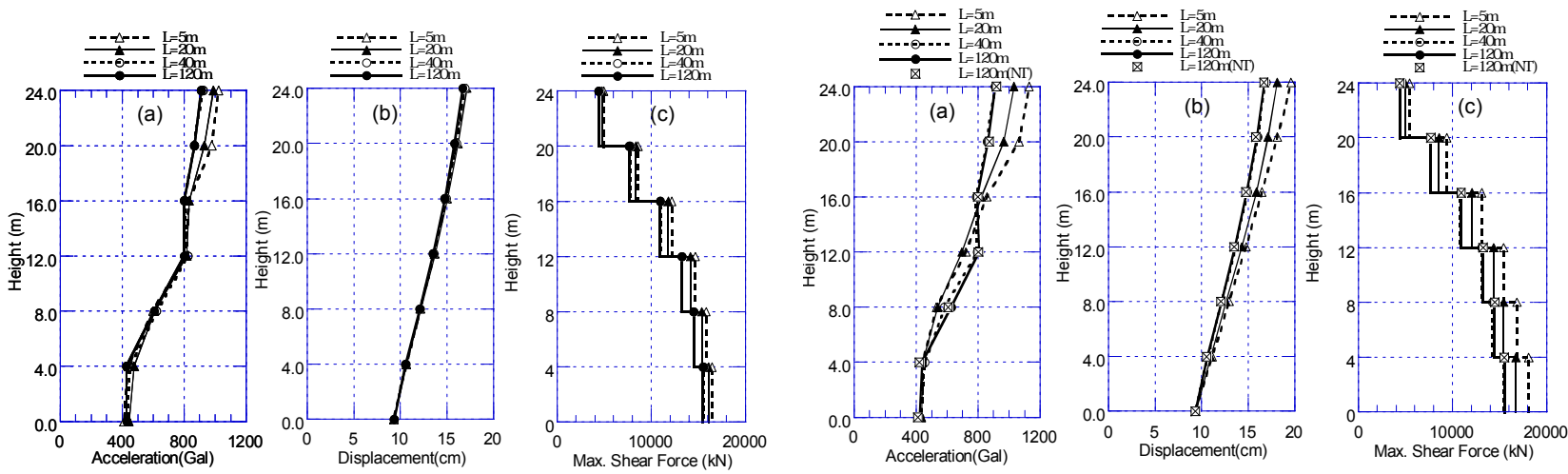

Figure 10 Comparison of maximum response value of the building (case NT: with nonlinear TB), (a) Maximum response acceleration, (b) Maximum response displacement, and (c) Maximum shear force.

Figure 11 Comparison of maximum response value of the building (case LT: with linear TB), (a) Maximum response acceleration, (b) Maximum response displacement, and (c) Maximum shear force
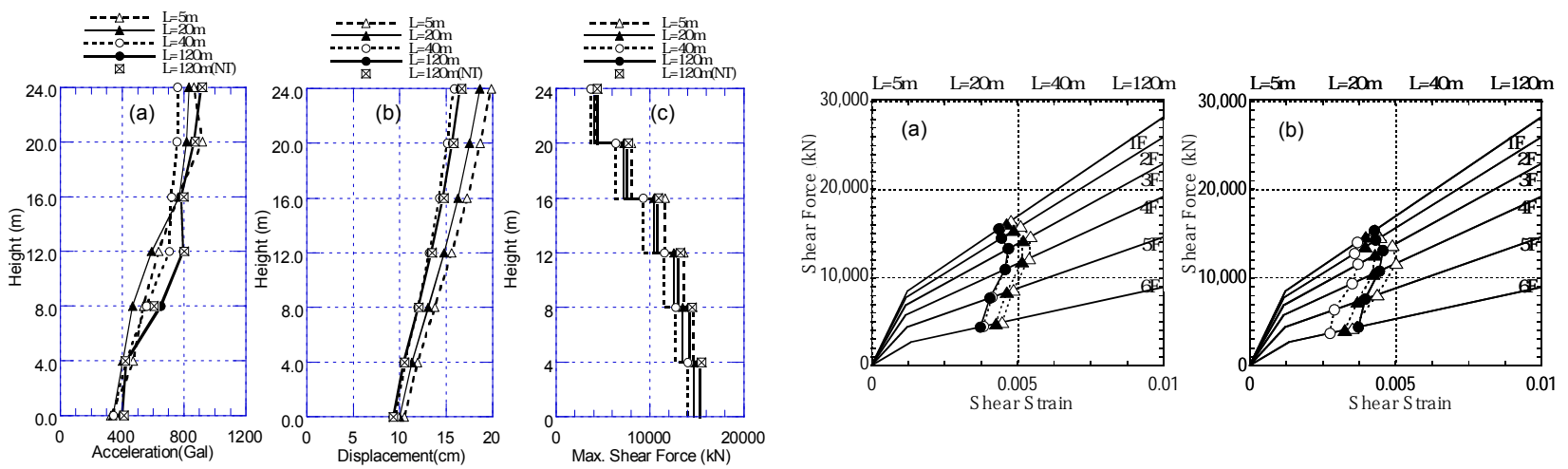

Figure 12 Comparison of maximum response value of the building (case V: with VB), (a) Maximum response acceleration, (b) Maximum response displacement, and (c) Maximum shear force.

Figure 13 Comparison of the maximum response value on the skeleton curve, (a) Case NT: with nonlinear TB, and (b) Case V: with VB

\subsection{Maximum response value of the building}

First, the results of the building response in each case are shown below. The response results using the nonlinear TB proposed in this paper are shown below. Figure 10 shows a comparison of the maximum response values (acceleration, displacement, and shear force) of the building for representative models $(\mathrm{L}=5 \mathrm{~m}, 20 \mathrm{~m}, 40 \mathrm{~m}, 120 \mathrm{~m})$. The line with black circles indicates the response result of $\mathrm{L}=120 \mathrm{~m}$ where the inner field is at a maximum and likely to be the most accurate. For all figures it can be said that all models correspond well with the most accurate result. This shows that the accuracy of the response results was good even if $\mathrm{L}$ is small. Accordingly, it can be said that the response results of this model have good accuracy even for a small model region.

Next, the response results using the equivalent linear TB proposed in the previous study are shown. Figure 11 shows a comparison of the maximum response values. The response re- 
sults of $\mathrm{L}=120 \mathrm{~m}$ for Case NT are also shown in the figure. In all the figures, the results of $\mathrm{L}=120 \mathrm{~m}$ for this case and are generally similar to the results of Case NT. Accordingly, the result of this case has slightly lower accuracy than Case NT, and it is necessary to make L greater than $40 \mathrm{~m}$ in order to make the differences less than $\pm 10 \%$.

Finally, the response results when using VB is shown, which is a representative method of conventional methods. Figure 12 shows a comparison of the maximum values of the building for each model. In all figures, the results of $\mathrm{L}=120 \mathrm{~m}$ are generally similar for Case $\mathrm{V}$ and Case NT. The results of each model have some differences with the results of $\mathrm{L}=120 \mathrm{~m}$, and the variation is even greater compared to Figure 10 and Figure 11. In this case, unlike the Case LT, the increase of L does not improve the accuracy except when L is sufficiently large.

\subsection{Maximum response value on the skeleton curve}

Figure 13 compares the maximum response value on the skeleton curve of each floor, in the cases using the nonlinear TB (Case NT) and VB (Case V). The analysis results of $\mathrm{L}=5 \mathrm{~m}$, $20 \mathrm{~m}, 40 \mathrm{~m}$, and $120 \mathrm{~m}$ are plotted. Results of $\mathrm{L}=120 \mathrm{~m}$ (line with black circles) are almost the same at the nonlinear $\mathrm{TB}$ and $\mathrm{VB}$. Response value of $\mathrm{L}=5-40 \mathrm{~m}$ correspond relatively well to $\mathrm{L}=120 \mathrm{~m}$ at the nonlinear TB, but the difference is large in VB. Both of these results show a similar trend to that of the previous section.

\subsection{Comparison of calculation load}

All the analyses in this chapter were calculated using IBM Power5+ $(2.2 \mathrm{GHz})$. Compared to $\mathrm{VB}$, there are a lot of calculation procedures for calculation of $\mathrm{TB}$, so computation time is longer for the same model. However, as shown in the previous chapters, analysis accuracy is high so reduction of the inner field becomes possible.

In figures 10 to 12 , when the differences in response is under $10 \%, \mathrm{~L}=30 \mathrm{~m}$ is minimum in the nonlinear TB cases, $\mathrm{L}=40 \mathrm{~m}$ is minimum in the equivalent linear TB cases, and $\mathrm{L}=100 \mathrm{~m}$ in the VB cases. Computation times including pre-processing are 17.4 minutes $(13.8+3.6$ minutes), 16.6 minutes (16.3 +0.3 minutes), and 17.2 minutes respectively. There are no significant differences in this problem. However, in 3-dimensional problems, due to the reduction of the analysis region, the reduction of computational load becomes noticeable, so this proposed method is considered to have a significant advantage.

\section{SUMMARY}

In the previous study, the author proposed the time history response analysis using the time domain energy TB while it was conventionally possible to apply to only the frequency domain. Accordingly, it became possible to consider nonlinear characteristics for the inner field. However, the outer field and TB were dealt as linear or equivalent linear. In this paper, by providing a method that enable to consider the nonlinear characteristic of TB, a seismic response analysis method that considers the entire analysis model as nonlinear was proposed.

First, the analysis method used in this paper was outlined. Particularly, TB may change every time step, so the computational load becomes significant when recalculations are continually made. Therefore, the author demonstrated a method to interpolate TB calculated at a representative time.

As a further example study, using these nonlinear characteristic evaluation methods, the seismic response analysis of the SSI system was conducted and the effectiveness of using the nonlinear TB was shown. By comparison with $\mathrm{VB}$, which is a representative conventional method, it was shown that there was a big advantage in accuracy when using the nonlinear TB, especially in 3-dimensional problems. 


\section{REFERENCES}

[1] J. Lysmer, T. Udaka, H. B. Seed, R. N. Hwang, FLUSH a Computer Program for Approximate 3-D Analysis of Soil-structure Interaction Problems, Report No.EERC75-30, University of California, Berkeley, 1975

[2] J. Lysmer, T. Udaka, C.-F. Tsai, H. B. Seed, , ALUSH a Computer Program for Seismic Response Analysis of Axisymmetric Soil-structure Systems, Report No.EERC75-31, University of California, Berkeley, 1975

[3] N. Nakamura, A Practical Method to Transform Frequency Dependent Impedance to Time Domain, Earthquake Engineering and Structural Dynamics, 35, 217-231, 2006

[4] N. Nakamura, Improved Methods to Transform Frequency Dependent Complex Stiffness to Time Domain, Earthquake Engineering and Structural Dynamics, 35, 1037 1050,2006

[5] N. Nakamura, Nonlinear Response Analysis of Soil-Structure Interaction System Using Transformed Energy Transmitting Boundary in the Time Domain, Soil Dynamics and Earthquake Engineering, 29, 799-808, 2009

[6] J. Lysmer, R.L. Kuhlelameyer, Finite Dynamic Model for Infinite Area, J. Eng. Mech. Div., ASCE, 95, EM3, 859-877, 1969

[7] P. B. Schnabel, J. Lysmer, H. B. Seed, SHAKE A Computer Program for Earthquake Response Analysis of Horizontally Layered Sites, Report No.EERC72-12, University of California, Berkeley, 1972

[8] N. Nakamura, Practical Causal Hysteretic Damping, Earthquake Engineering and Structural Dynamics, 36, 597-617, 2007

[9] N. Nakamura, A Study on Application of Nonlinear Causal Hysteretic Damping Model to Multi-node System Soil Model, Response Analysis of Soil Deposit Considering Both Frequency and Strain Amplitude Dependencies Using Nonlinear Causal Hysteretic Damping Model, J. of Structural and Construction Engineering (Transaction of AIJ), 662, 107-114 (In Japanese), 2007 